Check for updates

Cite this: Phys. Chem. Chem. Phys., $2018,20,28634$

Received 21st June 2018 Accepted 25th October 2018

DOI: $10.1039 / \mathrm{c} 8 \mathrm{cp} 03934 \mathrm{~h}$

rsc.li/pccp

\title{
The interplay between single particle anisotropy and interparticle interactions in ensembles of magnetic nanoparticles $\dagger$
}

\author{
G. Muscas, (D) ${ }^{a b}$ G. Concas, (D) $c$ S. Laureti, ${ }^{a}$ A. M. Testa, (D) ${ }^{a}$ R. Mathieu, (D) ${ }^{d}$ \\ J. A. De Toro, (D) ${ }^{\text {e }}$ C. Cannas, (D) ${ }^{f}$ A. Musinu, ${ }^{f}$ M. A. Novak, ${ }^{g}$ C. Sangregorio, (D) ${ }^{h}$ \\ Su Seong Lee ${ }^{i}$ and D. Peddis (D) *a
}

\begin{abstract}
This paper aims to analyze the competition of single particle anisotropy and interparticle interactions in nanoparticle ensembles using a random anisotropy model. The model is first applied to ideal systems of non-interacting and strongly dipolar interacting ensembles of maghemite nanoparticles. The investigation is then extended to more complex systems of pure cobalt ferrite $\mathrm{CoFe}_{2} \mathrm{O}_{4}$ (CFO) and mixed cobalt-nickel ferrite $(\mathrm{Co}, \mathrm{Ni}) \mathrm{Fe}_{2} \mathrm{O}_{4}$ (CNFO) nanoparticles. Both samples were synthetized by the polyol process and exhibit the same particle size $\left(D_{\mathrm{TEM}} \approx 5 \mathrm{~nm}\right.$ ), but with different interparticle interaction strengths and single particle anisotropy. The implementation of the random anisotropy model allows investigation of the influence of single particle anisotropy and interparticle interactions, and sheds light on their complex interplay as well as on their individual contribution. This analysis is of fundamental importance in order to understand the physics of these systems and to develop technological applications based on concentrated magnetic nanoparticles, where single and collective behaviors coexist.
\end{abstract}

\section{Introduction}

Magnetic nanoparticles (NPs) are complex physical objects that are interesting from both fundamental ${ }^{1-4}$ and technological points of view (e.g., MRI, ${ }^{5}$ hyperthermia, ${ }^{6}$ drug delivery,${ }^{5,7}$ catalysis, $^{8}$ microwave applications $\left.{ }^{9}\right)$. For magnetic monodomain particles with uniaxial anisotropy and easy axis

\footnotetext{
${ }^{a}$ Istituto di Struttura della Materia - CNR, o0016 Monterotondo Scalo (RM), Italy. E-mail: davide.peddis@cnr.it

${ }^{b}$ Department of Physics and Astronomy, Uppsala University, Box 516, SE-751 20 Uppsala, Sweden. E-mail: giuseppe.muscas@physics.uu.se

${ }^{c}$ Dipartimento di Fisica, Università di Cagliari, S. P. Monserrato-Sestu km 0.700, 09042 Monserrato, Italy

${ }^{d}$ Department of Engineering Sciences, Uppsala University, Box 534, SE-751 21 Uppsala, Sweden

${ }^{e}$ Instituto Regional de Investigación Cientifica Aplicada (IRICA), Departamento de Fisica Aplicada, Universidad de Castilla-La Mancha - 13071 Ciudad Real, Spain

${ }^{f}$ Dipartimento di Scienze Chimiche e Geologiche, INSTM, Università di Cagliari,

S. P. Monserrato-Sestu km 0.700, 09042 Monserrato, Italy

${ }^{g}$ Instituto de Fisica, Universidade Federal do Rio de Janeiro, Rio de Janeiro RJ 21945-970, Brazil

${ }^{h}$ Istituto di Chimica dei Composti Organo-Metallici, Consiglio Nazionale delle Ricerche and INSTM via Madonna del Piano 10, I-50019 Sesto Fiorentino, Italy ${ }^{i}$ Institute of Bioengineering and Nanotechnology, 31 Biopolis Way, The Nanos, Singapore 138669, Singapore

$\dagger$ Electronic supplementary information (ESI) available. See DOI: 10.1039/ c8cp03934h
}

alignment in the direction of the external field $\mu_{0} H$, the energy can be written as:

$$
E=K_{\mathrm{a}} V_{\mathrm{p}} \sin ^{2} \vartheta-M_{\mathrm{S}} V_{\mathrm{p}} \mu_{0} H \cos (\varphi-\vartheta)
$$

where $K_{\mathrm{a}}$ is the effective anisotropy constant, $V_{\mathrm{p}}$ is the particle volume, $M_{\mathrm{S}}$ is the saturation magnetization, and $\vartheta$ and $\varphi$ are, respectively, the angles between the magnetization vector and the magnetic field relative to the easy direction. ${ }^{10,11}$ Above a specific temperature, i.e., the blocking temperature $T_{\mathrm{B}}$, each particle behaves as a paramagnetic superspin, hence with dynamics defined in the framework of superparamagnetism (SPM). ${ }^{12}$ The design of nanoparticle-based magnetic materials with specific properties hinges on the control of the energy profile of individual NPs, which is proportional to $V_{\mathrm{p}}$ and $K_{\mathrm{a}}$, where the effective anisotropy constant mainly depends on the particle crystalline structure, chemical composition, and morphology. In addition, upon reducing the particle size, the increasing surface-to-volume ratio can strongly influence the magnetic anisotropy (i.e., surface anisotropy). ${ }^{13}$ The magnetic behavior of a nanoparticle ensemble is also strongly affected by interparticle interactions that can be dipole-dipole or based on exchange coupling between surface atoms. ${ }^{14,15}$ The nature and strength of interparticle interactions depend on the nanoparticles' arrangement, ${ }^{13}$ and on the presence of non-magnetic spacers (e.g., organic ligands, silica shell). For example, a dipolar interaction may yield a dynamical magnetic frustration and a superspin 
glass (SSG) state. Such a collective state exhibits similar dynamical features to atomic spin glasses, e.g., aging and rejuvenation. ${ }^{16}$ Further enhancement of the interactions leads to an ordered collective organization of superspins defined as superferromagnetism (SFM). ${ }^{17}$ Evidence of such a state has been provided experimentally on granular films and discussed theoretically in terms of an effective mean-field model. ${ }^{18}$ Strong interactions can induce a hysteretic behavior even above the intrinsic blocking temperature of the nanoparticles, clearly visible in the discontinuity of the thermal dependence of the coercive field. In addition, in the case of particles with broad and/or multiple size distribution, a multitude of magnetic states can emerge. Upon cooling the system, the progressive "blocking" from the largest to the smallest particles can induce a progressive shift from a pure SPM to an SSG state and finally a complete chainlike SFM ordering even for purely dipolar interacting particles. ${ }^{19} \mathrm{It}$ should be underlined that concentrated ensembles of individually responding magnetic entities are required for several applications (e.g., magnetic recording, drug delivery, magnetic hyperthermia), where interparticle interactions cannot be neglected. ${ }^{20}$ In this context, the magnetic behavior of an ensemble of nanoparticles can be described as a complex interplay between the single particle anisotropy (SPA) and the interparticle interactions (II). The effect of $\mathrm{SPA}^{12,21-24}$ and $\mathrm{II}^{20,25-27}$ on the magnetic properties of the nanoparticles has been thoroughly discussed in the literature. Nevertheless, a clear approach to separate and quantify the individual contribution of SPA and II in the dense ensembles of the nanoparticles is still lacking, since, due to the overlapping of the two contributions, the collective order is usually hidden by the SPM blocking behavior. ${ }^{28,29}$

The magnetic properties of ferromagnetic materials are strongly connected to their crystalline structures, which determine their magneto-crystalline anisotropy and their symmetry axis. For typical (non-textured) nanoparticle ensembles and polycrystalline continuous media, the easy axes of the constituent grains/particles are randomly oriented. In polycrystalline materials, the exchange interactions force the spins to align over a correlation length $\left(L_{\text {corr }}\right)$, which can be larger than the grain size $(D)$. Therefore, the effective anisotropy for the correlated volume will be the average of the grains within such a volume, resulting in a reduced magnitude with respect to the anisotropy of the individual grain. ${ }^{30}$ Within this context, the Random Anisotropy Model (RAM) has been employed to describe systems where the exchange interactions dominate, extending the correlation volume beyond the structural correlation length, i.e., the grain size $D$. The RAM has been extensively used for describing amorphous materials ${ }^{31}$ since the 1970s, and more recently it has been also used for nano-crystalline metal alloys. ${ }^{32}$ In addition, a modified RAM has been proposed to explain the properties of $\mathrm{Co}_{x}\left(\mathrm{SiO}_{2}\right)_{1-x}$ nanogranular films, ${ }^{33,34}$ suggesting that, with opportune modifications, this phenomenological model can be generally applied regardless of the nature of the interactions, exchange or dipolar, between particles. ${ }^{30}$ Indeed, the same model was able to describe the field dependence of the blocking temperature of 2D arrays of iron oxide nanoparticles deposited as monolayers. ${ }^{35}$
In the present paper, we propose the application of the RAM to analyze the magnetic properties of strongly interacting nanoparticle ensembles, extending the model for the first time to the pure dipolar interaction case. The effective anisotropy energy of such systems was deeply investigated, identifying quantitatively the individual contribution of the single particle anisotropy and interparticle interaction energies. Specific nanoparticle systems were selected to test the RAM. In the first stage, the influence of interparticle interactions in ensembles of maghemite nanoparticles was analyzed by comparing the magnetic properties of the nanoparticles isolated by a thick silica coating with a packed ensemble of the same particles. Then, the investigation was extended to more complex ensembles of $(\mathrm{Co}, \mathrm{Ni}) \mathrm{Fe}_{2} \mathrm{O}_{4}$ nanoparticles, with the same size but with different interaction strength and single particle anisotropy.

\section{Random anisotropy model (RAM)}

For ferromagnetic materials, the exchange interaction energy starts to balance the anisotropy at a specific length scale defined as:

$$
L_{0}=\varphi_{0} \sqrt{\frac{A_{0}}{K_{\mathrm{a}}}}
$$

$L_{0}$ represents the ferromagnetic correlation length, while $\varphi_{0}$ is a proportionality constant close to $1, A_{0}$ is the exchange stiffness and $K_{\mathrm{a}}$ is the anisotropy constant. This length scale describes the distance within which no significant variation in the magnetization direction is observed, and it usually extends over a few tens of $\mathrm{nm}\left(e . g\right.$., $L_{0} \approx 5-10 \mathrm{~nm}$ for Co-based alloys and $L_{0} \approx 20-40 \mathrm{~nm}$ for Fe-based alloys). ${ }^{30}$ For traditional polycrystalline materials, the crystallite size $D$ is much larger than $L_{0}$, thus the magnetization reversal is dominated by the magnetocrystalline easy axis. On the other hand, for systems where $D \ll L_{0}$, the magnetization is forced to be aligned over a region where the local easy axes are distributed in different directions. ${ }^{30,36}$ In the case of ensembles of dipolar interacting nanoparticles, we define a more general magnetic correlation length $\left(L_{\text {corr }}\right)^{37}$ that can be expressed as a function of the applied field: ${ }^{32,34}$

$$
L_{\mathrm{corr}}=D+\left[\frac{2 A_{\mathrm{eff}}}{M_{\mathrm{S}} \mu_{0} H+C}\right]^{1 / 2}
$$

$A_{\text {eff }}$ represents the interaction intensity, ${ }^{30}$ which for nanocrystalline alloys is the inter-grain exchange constant $A .^{33}$ The parameter $C$ was introduced for the nanoparticle systems to overcome the divergence at $\mu_{0} H=0 \mathrm{~T}$. It should take into account the influence of particle concentration on the interactions, assuming a value close to zero for clustered particles and the form $C \approx 2 A_{\text {eff }}-M_{\mathrm{S}} \mu_{0} H$ for non-interacting particles. $^{34,35}$

$L_{\text {corr }}$ defines a correlation volume $\left(V_{\mathrm{N}}\right)$, where the effective anisotropy constant $K_{\text {eff }}$ results from the averaging over the 


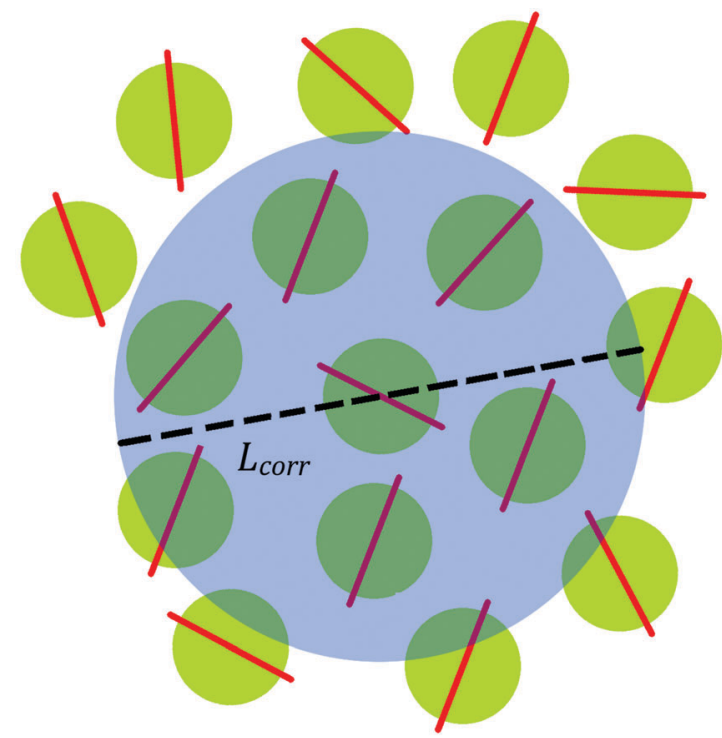

Fig. 1 Sketch of an ensemble of interacting nanoparticles. The blue circle represents the correlation volume $V_{N}$ described by the correlation length $L_{\text {corr }}$ (dashed black line). Within such a volume, the average anisotropy is mediated among the different particles due to the random distribution of their individual anisotropy axes (red lines).

number of particles $(N)$ within such a volume, ${ }^{38}$ as described by the sketch in Fig. 1, as reported in ref. 30:

$$
\left\langle K_{\mathrm{eff}}\right\rangle=\frac{K_{\mathrm{a}}}{\sqrt{N}}
$$

The number of correlated particles $N$ can be defined as the ratio between the correlation volume and the volume of a single particle, taking into account the volume fraction $x$ that the particles occupy in the ensemble:

$$
N=1+x \frac{\left(L_{\text {corr }^{3}}-D^{3}\right)}{D^{3}}
$$

Therefore, we can define the volume of the cluster $V_{\mathrm{N}}$ as the effective volume of the magnetic material interacting within the correlation length $L_{\text {corr }}$ :

$$
V_{\mathrm{N}}=\frac{\pi}{6}\left[D^{3}+x\left(L_{\mathrm{corr}}^{3}-D^{3}\right)\right]
$$

As the interparticle interactions increase, the correlation length expands and the anisotropy is mediated within a larger number of particles, thus reducing its effective magnitude. On the other hand, the action of an external magnetic field reduces the correlation length ${ }^{32}$ and prevents the "cluster" formation. It is worth mentioning that the expressions (4) and (6) tend to the anisotropy and volume of an individual particle when the interactions are very weak and $L_{\text {corr }} \rightarrow D$.

For a non-interacting system, the dependence of the superparamagnetic blocking temperature from the field $\mu_{0} H$ is described by the law: ${ }^{34,35}$

$$
T_{\mathrm{B}}=\frac{K_{\mathrm{a}} V_{\mathrm{p}}}{k_{\mathrm{B}} \ln \left(\frac{\tau_{\mathrm{m}}}{\tau_{0}}\right)}\left[1-\frac{\mu_{0} H}{\mu_{0} H_{\mathrm{K}}}\right]^{\alpha}
$$

with $\alpha=1.5^{35}$ and $\tau_{\mathrm{m}}=60 \mathrm{~s}$, i.e., the typical experimental time in $\mathrm{dc}$ magnetization measurements, $\tau_{0}=10^{-11} \mathrm{~s}$ for the ferromagnetic particles and the Boltzmann constant $k_{\mathrm{B}}=1.38065 \times$ $10^{-23} \mathrm{~J} \mathrm{~K}^{-1}$. The anisotropy constant $K_{\mathrm{a}}$ can be evaluated using the relation:

$$
K_{\mathrm{a}}=\frac{M_{\mathrm{S}} \mu_{0} H_{\mathrm{K}}}{2}
$$

where $\mu_{0} H_{\mathrm{K}}$ is the anisotropy field. According to the RAM, for an ensemble of interacting particles, one should consider the effective anisotropy field $\mu_{0} H_{\mathrm{K}}^{N}$ of the cluster of $N$ correlated particles, which is linked to its effective average anisotropy $K_{\text {eff: }}{ }^{34,35}$

$$
\mu_{0} H_{\mathrm{K}}^{N}=2 \frac{K_{\mathrm{eff}}}{M_{\mathrm{S}}}
$$

Finally, eqn (7) can be re-written by substituting $K_{\mathrm{a}}, V_{\mathrm{p}}$ and $H_{\mathrm{K}}$ with the effective values for the clusters $K_{\mathrm{eff}}, V_{\mathrm{N}}$ and $H_{\mathrm{K}}^{N}$, respectively:

$$
T^{*}(H)=\frac{K_{\mathrm{eff}} \mathrm{V}_{\mathrm{N}}}{k_{\mathrm{B}} \ln \left(\frac{\tau_{\mathrm{m}}}{\tau_{0}}\right)}\left[1-\frac{\mu_{0} H}{\mu_{0} H_{\mathrm{K}}^{N}}\right]^{1.5}
$$

Note that another expression for $T^{*}(H)$ has been recently considered. ${ }^{39,40}$ The experimental values of $T^{*}(H)$ can be extracted from the temperature dependence of the difference between field-cooled and zero-field-cooled curves $\left(M_{\mathrm{FC}}-M_{\mathrm{ZFC}}\right)$. For non-interacting particles, an estimate of the distribution of the anisotropy energy barrier can be obtained from:

$$
f(T) \propto \frac{\mathrm{d}\left(M_{\mathrm{ZFC}}-M_{\mathrm{FC}}\right)}{\mathrm{d} T}
$$

Because of interparticle interactions, eqn (11) gives only a rough indication of the distribution of anisotropy energies. As an example, the $\left(M_{\mathrm{FC}}-M_{\mathrm{ZFC}}\right)$ curve and its negative derivative are reported in ESI $\dagger$ in Fig. S1 for sample S17. Within the Néel model, the blocking temperature $T_{\mathrm{B}}$ can be considered as the temperature for which the relaxation time is equal to the measuring time of the experimental technique. In a real system of nanoparticles, where a finite size distribution always exists, $T_{\mathrm{B}}$ is often defined as the temperature at which $50 \%$ of the sample is in the superparamagnetic state ${ }^{41,42}$ Since the blocking temperature of a single particle is proportional to its anisotropy barrier, an estimate of $T_{\mathrm{B}}$, i.e., $T^{*}$, can be obtained as the temperature at which the integral of $\mathrm{d}\left(M_{\mathrm{ZFC}}-M_{\mathrm{FC}}\right) / \mathrm{d} T$ reaches $50 \%$ of its maximum value, i.e., the temperature at which $\left(M_{\mathrm{FC}}-M_{\mathrm{ZFC}}\right)$ is reduced to half of its value at the lowest temperature.

The experimental $T^{*}(H)$ values can be fitted according to eqn (10). By combining eqn (3)-(6), and (8), one needs as input parameters the experimental value of $M_{\mathrm{S}}$, the particle size $D$ and the volume fraction $x$. The only free parameters of the fit are the effective interaction strength $A_{\text {eff }}$, the parameter $C$ and the intrinsic anisotropy constant of the magnetic material $K_{\mathrm{a}}$. Finally, by using such values in eqn (3)-(5), and (6), further 
information about the cluster and the individual particles can be obtained.

\section{Results and discussion}

\subsection{Effect of interparticle interactions}

When magnetic nanoparticles are arranged in concentrated ensembles, their magnetic behavior is strongly affected by the interparticle interactions. Recently, De Toro et al. showed how strongly interacting nanoparticles may create a "nanoparticle replica of the spin-glass state". ${ }^{25,43}$ They produced a packed disc of maghemite NPs with average size $\approx 8 \mathrm{~nm}$ and very narrow size distribution by pressing a mass of about $100 \mathrm{mg}$ of powders at approximately $0.7 \mathrm{GPa}$. This sample (labelled RCP8) was compared with another one where the same particles were coated by an $\approx 17 \mathrm{~nm}$ thick silica shell (labelled S17), in order to increase the interparticle distance and reduce the effect of interactions to a negligible value. The volume-filling factor of the magnetic phase has been determined by Archimedes' method as $\approx 67 \%$ and $\approx 0.4 \%$ for RCP8 and S17, respectively. Fig. 2a shows the $M_{\mathrm{ZFC}}$ and $M_{\mathrm{FC}}$ curves of the two samples. The $M_{\mathrm{ZFC}}$ curves show a maximum at a temperature $\left(T_{\max }\right)$ that, for non-interacting particles such as for $\mathrm{S} 17$, is directly proportional to the average blocking temperature, with a proportionality constant of $\beta=1.5-2$, depending on the type of particle size distribution. ${ }^{44}$ An irreversible magnetic behavior is observed below a given temperature ( $\left.T_{\mathrm{irr}}\right)$ that corresponds to the blocking of the biggest particles. S17 exhibits the typical features of the isolated particle systems, such that an FC magnetization shows a Curie-like behavior. On the other hand, the dense packing of the particles in RCP8 modifies the original superparamagnetic behavior of the isolated particles. $T_{\max }$ exhibits a much larger value, in addition, the FC curves flatten out below $T_{\max }$, both being typical features of strong interparticle interactions, which induce a superspin-glass state in the sample. ${ }^{25}$ Within this picture, we propose a modified random anisotropy model as a way to explain the origin of such a collective state.

At small cooling fields, for the non-interacting sample S17, $T_{\mathrm{B}} \approx T^{*}$, which corresponds to a blocking temperature $\approx 22 \mathrm{~K}$. On the other hand, sample RCP8 is a strongly interacting sample with $T^{*} \approx 77 \mathrm{~K}$. Due to such strong interparticle interactions, responsible of a collective behavior, $T_{\mathrm{B}} \approx T^{*}$ is not more valid, thus it is not possible to get the information about the blocking temperature and the anisotropy energy of individual particles of this sample. Further analysis was carried out for both $\mathrm{S} 17$ and RCP8 samples, measuring $\left(M_{\mathrm{FC}}-M_{\mathrm{ZFC}}\right)$
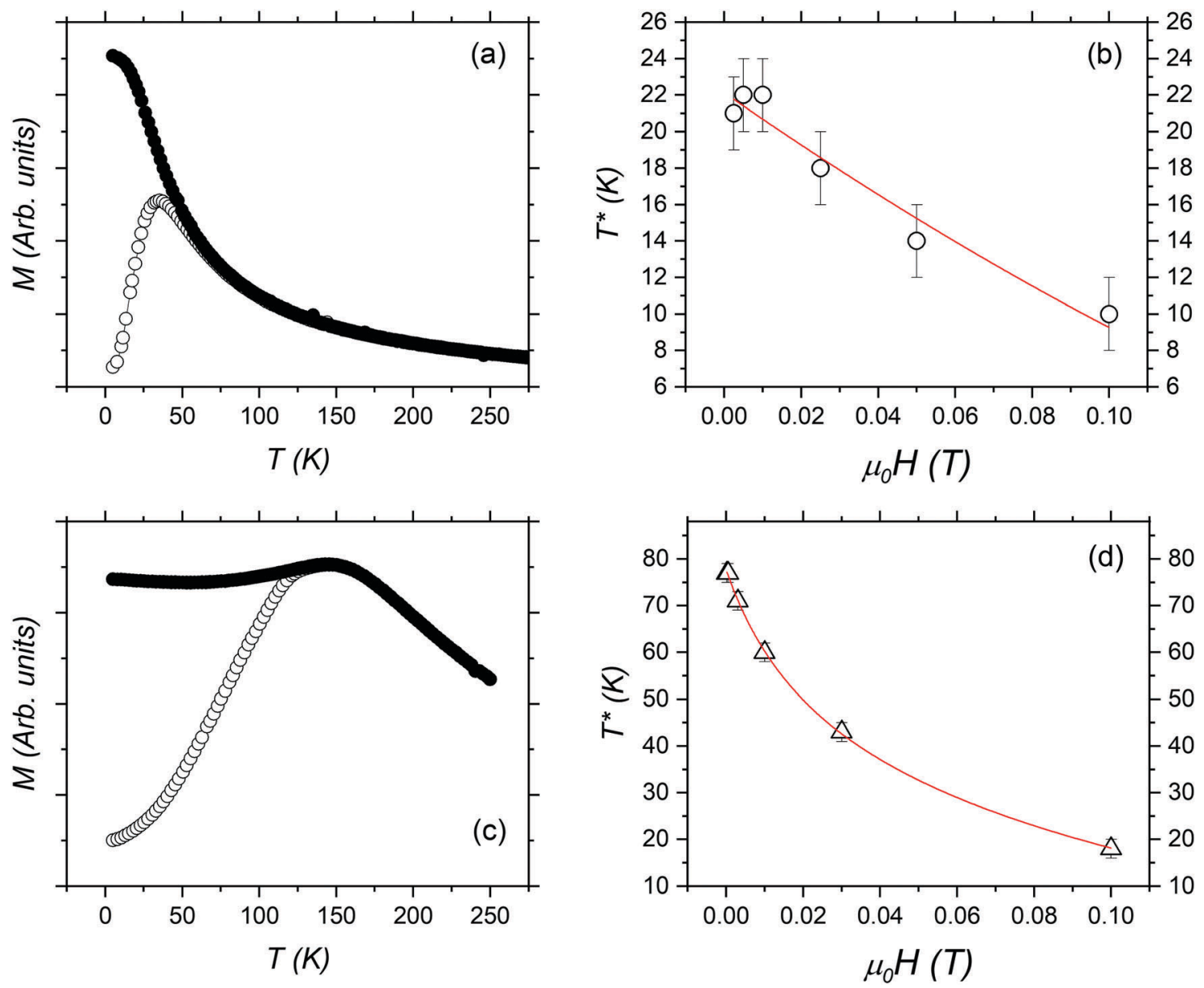

Fig. $2 M_{\mathrm{ZFC}}$ (empty symbols) and $M_{\mathrm{FC}}$ (full symbols) curves measured at applied fields of $2.5 \mathrm{mT}$ and $3.0 \mathrm{mT}$ for S17 (a) and RCP8 (c), respectively. The $T^{\star}$ vs. field curves (dots) and the RAM fit (red line) for samples S17 (b) and RCP8 (d). Uncertainties are reported as bars. 
Table 1 The anisotropy constant $K_{\mathrm{a}}$ and the correlation length $L_{\text {corr }}$ obtained from the RAM fit are reported here along with the saturation magnetization $M_{\mathrm{S}}$, experimentally obtained from the $M(H)$ loop and used as an input parameter in the fit. $T_{\mathrm{B}}$ was estimated as $T^{\star}$ for $\mathrm{S} 17$ and extracted from the RAM fit in the limit of $\mu_{0} H=0$ T for RCP8. For S17, $L_{\text {corr }}$ corresponds to the particle's diameter within the estimated error, confirming the non-interacting behavior of the sample

\begin{tabular}{lllll}
\hline Sample & $K_{\mathrm{a}}\left(\mathrm{J} \mathrm{m}^{-3}\right)$ & $L_{\text {corr }}(\mathrm{nm})$ & $T_{\mathrm{B}}(\mathrm{K})$ & $M_{\mathrm{S}}\left(\mathrm{A} \mathrm{m}^{-1}\right)$ \\
\hline S17 & $34(5) \times 10^{3}$ & $8.0(5)$ & $22(4)$ & $225(1) \times 10^{3}$ \\
RCP8 & $41(5) \times 10^{3}$ & $18.2(1)$ & $31(4)$ & $225(1) \times 10^{3}$
\end{tabular}

with different cooling fields. The values of $T^{*} v s$. the applied field were fitted according to eqn (10), as shown in Fig. 2 with data summarized in Table 1. For the isolated (i.e., noninteracting) particles in S17, De Toro et al. measured a value of $K_{\mathrm{a}} \approx 30 \times 10^{3} \mathrm{~J} \mathrm{~m}^{-3}$ by means of AC susceptibility data. ${ }^{25}$ The $K_{\mathrm{a}}$ value extracted from the RAM fitting is $34(5) \times 10^{3} \mathrm{~J} \mathrm{~m}^{-3}$, in good agreement with the AC results. Furthermore, the same procedure applied to RCP8 allowed the estimation of the intrinsic anisotropy constant of the single non-interacting particles as $41(5) \times 10^{3} \mathrm{~J} \mathrm{~m}^{-3}$, in agreement, within the experimental error, with the previous values (Table 1). This confirms that the RAM analysis allows determination of the correct value of the anisotropy constant even in a strongly interacting system, where the blocking temperature of the individual NPs is hidden by the collective behavior. Note that demagnetization effects influence the $M_{\mathrm{ZFC}}$ and $M_{\mathrm{FC}}$ curves for RCP8.$^{45}$ Anyway, we have verified that this does not affect the $T^{*}$ determination beyond the experimental error. Furthermore, the single particle $T_{\mathrm{B}}$ can be calculated from (10) for the case of $\mu_{0} H=0 \mathrm{~T}$, leading for sample RCP8 to an intrinsic single particle value of $T_{\mathrm{B}}=31(4) \mathrm{K}$, compatible with the experimental data of $22(4) \mathrm{K}$ of $\mathrm{S} 17$ for isolated-like particles.

\subsection{Single particle magnetic anisotropy and interparticle interactions}

The very flexible crystal chemistry of spinel ferrites was exploited to prepare a further model system to verify the application of the RAM on ensembles of strongly interacting nanoparticles. Spinel ferrites possess an FCC lattice of oxygen ions with $\mathrm{Fe}^{3+}$ and $\mathrm{Me}^{2+}$ cations, where Me denotes a divalent transition metal ion, distributed among octahedral $\left(O_{\mathrm{h}}\right)$ and tetrahedral $\left(T_{\mathrm{d}}\right)$ interstitial sites. The superexchange magnetic coupling between the cations located in the same site produces a parallel ferromagnetic alignment of spins, but the coupling between the two sub-lattices produces an antiparallel arrangement. Due to the different magnetic moment of the two lattices, a net magnetization emerges, producing a final ferrimagnetic ordering. ${ }^{20}$ Hence, the specific magnetic nature of divalent cations $\left(\mathrm{Ni}^{2+}, \mathrm{Fe}^{2+}, \mathrm{Co}^{2+}\right.$, and $\left.\mathrm{Mn}^{2+}\right)$ allows tuning the saturation magnetization and, most of all, the magnetic anisotropy, beyond the effect of particle size. ${ }^{21,22}$

Two ensembles of ferrite nanoparticles with the same average size of $\sim 4.5 \mathrm{~nm}$ (Fig. S3, ESI $\dagger$ ) were synthetized by the polyol process. A pure $\mathrm{CoFe}_{2} \mathrm{O}_{4}$ sample (CFO) has been compared with the one in which about $50 \%$ of cobalt was substituted with nickel (CNFO, $(\mathrm{CoNi}) \mathrm{Fe}_{2} \mathrm{O}_{4}$ ). Both samples represent concentrated systems with volume-filling factors of $52 \%$ and $39 \%$ for CFO and CNFO, respectively. Their particles are in close proximity, but not in contact since they are coated by polyol (see Section 2.2 and Fig. S4 in ESI $\dagger$ ) preventing possible super-exchange interactions. ${ }^{46} \mathrm{~A}$ detailed description of the samples is provided in the ESI $\dagger$ and in ref. 23.

The $M v s$. $H$ curves of the two samples recorded at $5 \mathrm{~K}$ are reported in Fig. 3a. By reducing the amount of cobalt, the magnetic anisotropy decreases, as reflected by a decrease in the coercive field $\mu_{0} H_{\mathrm{C}}$ and the saturation field $\mu_{0} H_{\text {sat }}$ (Table 2). The latter parameter is defined as the minimum field that
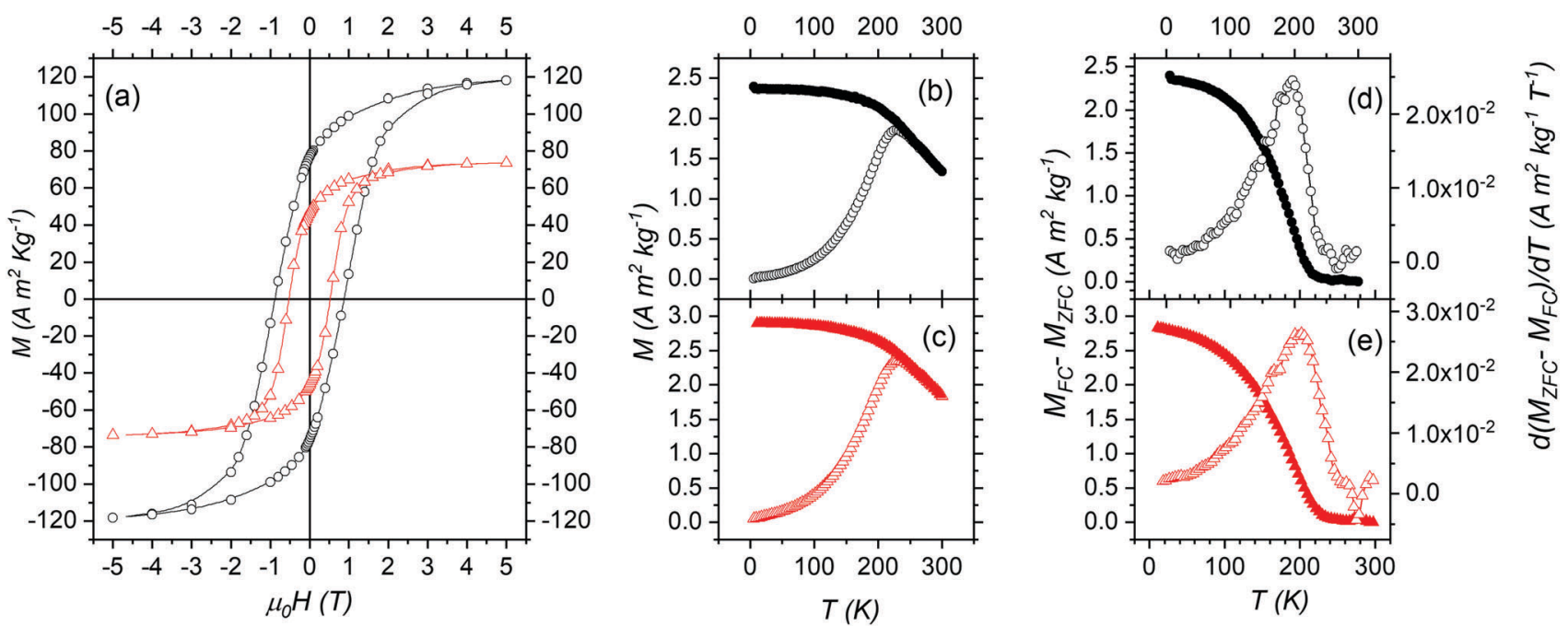

Fig. 3 Magnetization vs. applied field at $5 \mathrm{~K}$ (a) for samples CFO (black circles) and CNFO (red triangles). M $M_{\mathrm{ZFC}}$ (empty symbols) and $M_{\mathrm{FC}}$ (full symbols) for samples CFO (b) and CNFO (c). ( $M_{F C}-M_{Z F C}$ ) curves and their negative derivatives, represented as full and empty symbols, respectively, for samples CFO (d) and CNFO (e). 
Table 2 For each sample, the chemical composition according to the cation concentration measured by ICP analysis, the mean diameter $\left\langle D_{\text {TEM }}\right\rangle$, the saturation magnetization $\left(M_{S}\right)$, the coercive field $\left(\mu_{0} H_{C}\right)$, and the saturation field $\left(\mu_{0} H_{\text {sat }}\right)$ extracted from the $M(H)$ loops measured at $5 \mathrm{~K}$ are summarized

\begin{tabular}{llllll}
\hline Sample & $\begin{array}{l}\text { Experimental } \\
\text { formula }\end{array}$ & $\begin{array}{l}\left\langle D_{\text {TEM }}\right\rangle \\
(\mathrm{nm})\end{array}$ & $\begin{array}{l}M_{\mathrm{S}} \\
\left(\mathrm{A} \mathrm{m}^{2} \mathrm{~kg}^{-1}\right)\end{array}$ & $\begin{array}{l}\mu_{0} H_{\mathrm{C}} \\
(\mathrm{T})\end{array}$ & $\begin{array}{l}\mu_{0} H_{\text {sat }} \\
(\mathrm{T})\end{array}$ \\
\hline CFO & $\mathrm{Co}_{0.97} \mathrm{Fe}_{2.00} \mathrm{O}_{4.00}$ & $4.5(1)$ & $130(10)$ & $0.88(7)$ & $3.1(8)$ \\
CNFO & $\mathrm{Co}_{0.40} \mathrm{Ni}_{0.58} \mathrm{Fe}_{2.00} \mathrm{O}_{4.00}$ & $4.6(1)$ & $77(4)$ & $0.50(7)$ & $2.4(3)$
\end{tabular}

reverses the moment of the particles with the highest anisotropy energy. It was evaluated as the field at which the difference between the two branches of the $M(H)$ curves was reduced down to $1 \%$ of their maximum value. ${ }^{23}$ The larger anisotropy energy of sample CFO can be ascribed to its higher Co content. Cobalt ions have a marked magneto-crystalline anisotropy far above that of nickel and iron, indeed its orbital magnetic moment is not quenched by the crystal field, with a particular strong spin-orbit coupling for $\mathrm{Co}^{2+}$ ions in octahedral sites. ${ }^{47-49}$ The difference in saturation magnetization among CFO and CNFO can be explained by the different magnetic moments of $\mathrm{Co}$ and $\mathrm{Ni}$ and an unusual cationic distribution, as discussed in ref. 23.

The thermal dependence of the magnetization measured by $\mathrm{ZFC}, \mathrm{FC}$ and $\left(M_{\mathrm{FC}}-M_{\mathrm{ZFC}}\right)$ analysis gives interesting information about the blocking process (Fig. $3 \mathrm{~b}-\mathrm{e}$ and Table 3 ). In this case, the susceptibility is lower than that of sample RCP8, as well as the packing fraction. This enforces the assumption that the demagnetization effects have limited influence on these samples. Although the $M(H)$ curves indicate a different magnetic anisotropy, the two samples show equal values of $T_{\text {irr }}$ and $T_{\max }$, within the experimental error. This scenario is substantially confirmed by the $\left(M_{\mathrm{FC}}-M_{\mathrm{ZFC}}\right)$ magnetization curves measured after a field cooling of $2.5 \mathrm{mT}$ (see Fig. 3d and e). For both samples, $\left(M_{\mathrm{FC}}-M_{\mathrm{ZFC}}\right)$ decreases with increasing temperature, as expected for an ensemble of magnetic monodomain particles. The $T^{*}$ values follow the same behavior of $T_{\max }$ and $T_{\text {irr }}$, suggesting similar blocking temperatures for both samples. On the other hand, the values of $T^{*}(H)$ show different field dependences. A further confirmation of the unexpected situation, i.e., different magnetic anisotropy and the same blocking temperature, is provided by Mössbauer spectroscopy carried out at $300 \mathrm{~K}$ (Fig. 4). Generally speaking, the total Mössbauer spectrum of an ensemble of magnetic mono-domain particles is typically represented by the superposition of magnetic (six lines) and quadrupole (two lines) patterns. These two components are due to particles with

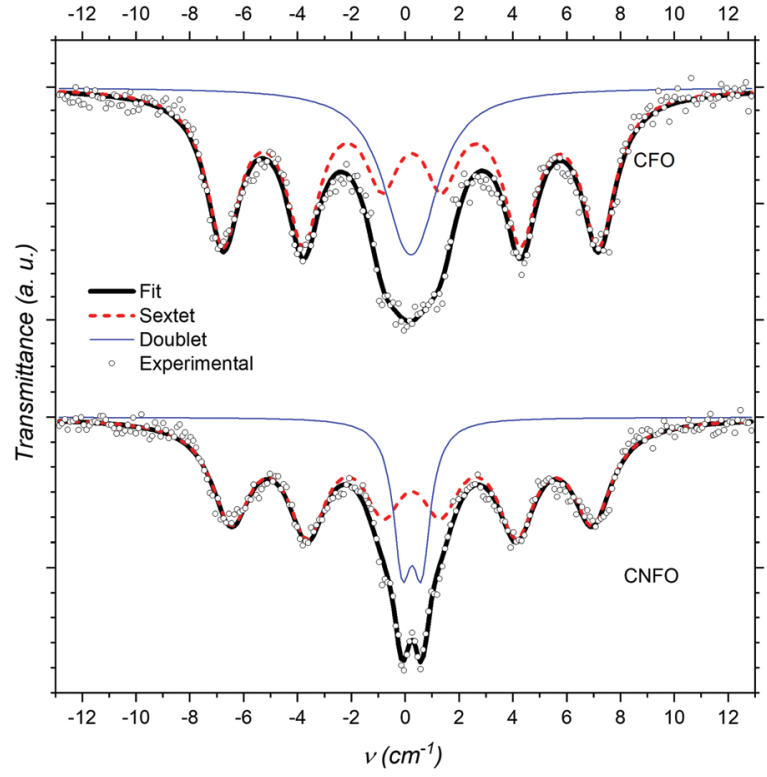

Fig. 4 Mössbauer measurements have been performed at room temperature with no applied external magnetic field. The experimental points are presented as empty small circles, the fit of the blocked fraction is represented by a dashed red line, and the superparamagnetic contribution by a blue thin line; the bold black line represents the complex fit.

superparamagnetic relaxation time that is, respectively, longer or shorter compared to the timescale of Mössbauer spectroscopy $\left(t_{\mathrm{w}}=5 \times 10^{-9} \mathrm{~s}\right)$. In the samples under investigation, the fraction of particles being superparamagnetic at room temperature is similar, actually slightly higher for CNFO with respect to $\mathrm{CFO}$ ( $\approx 82 v s$. $75 \%$, respectively). This result confirms the landscape drawn by $M v s$. $T$ measurements in low field, indicating that despite having different magnetic anisotropies, the superparamagnetic relaxation times of the two samples are very similar, as observed by the different time windows of two experimental methods.

In order to investigate the interaction regimes among the particles, the remanent magnetization was measured following the IRM (Isothermal Remanent Magnetization) and DCD (Direct Current Demagnetization) protocols (see Section 3 of ESI $\dagger$ for details). For non-interacting single-domain particles with uniaxial anisotropy and magnetization reversal by coherent rotation, the two remanence curves are related via the Wohlfarth equation ${ }^{50} m_{\mathrm{DCD}}(H)=1-2 m_{\mathrm{IRM}}(H)$, where $m_{\mathrm{DCD}}(H)$ and $m_{\mathrm{IRM}}(H)$ represent the reduced terms $M_{\mathrm{DCD}}(H) / M_{\mathrm{DCD}(5 \mathrm{~T})}$ and $M_{\mathrm{IRM}}(H) / M_{\mathrm{IRM}(5 \mathrm{~T})}$, with $M_{\mathrm{DCD}(5 \mathrm{~T})}$ and $M_{\mathrm{IRM}(5 \mathrm{~T})}$ being the remanence values for the DCD and IRM curves for a reversal

Table $3 T_{\max }$ and $T_{\text {irr }}$ values estimated from ZFC-FC curves and $T^{*}$ calculated from the $\mathrm{d}\left(\mathrm{M}_{\mathrm{ZFC}}-M_{\mathrm{FC}}\right) / \mathrm{d} T$ curve measured at $\mu_{0} H=2.5 \mathrm{mT}$. The linear distance which encloses two nanoparticles, considering also the surfactant $\left(L_{2 \mathrm{NP}}\right)$, is compared to the correlation length $\left(L_{\text {corr }}\right)$ calculated with the data obtained from the RAM fits with applied fields of $0 \mathrm{mT}$ and $2.5 \mathrm{mT}$. In addition, for CNFO, the maximum field $\left(\mu_{0} H_{\text {limit }}\right)$ that produces a correlation length sufficient to involve two entire particles in the correlation volume is shown

\begin{tabular}{llllllll}
\hline Sample & $T_{\max }(\mathrm{K})$ & $T_{\text {irr }}(\mathrm{K})$ & $T^{*}(\mathrm{~K})$ & $L_{2 \mathrm{NP}}$ & $L_{\text {corr }}\left(\mu_{0} H=0 \mathrm{~T}\right)$ & $L_{\text {corr }}\left(\mu_{0} H=2.5 \mathrm{mT}\right)$ & $\mu_{0} H_{\text {limit }}$ \\
\hline CFO & $229(4)$ & $255(4)$ & $174(2)$ & $10.1(1) \mathrm{nm}$ & $8.4(1) \mathrm{nm}$ & $8.3(1) \mathrm{nm}$ & - \\
CNFO & $231(4)$ & $255(4)$ & $170(2)$ & $10.8(1) \mathrm{nm}$ & $15.1(1) \mathrm{nm}$ & $14.8(1) \mathrm{nm}$
\end{tabular}


field of $5 \mathrm{~T}$, respectively. Kelly et $a .^{51}$ rewrote the Wohlfarth equation to explicitly reveal the deviations from a noninteracting case: ${ }^{51}$

$$
\Delta M=m_{\mathrm{DCD}}(H)-1+2 m_{\mathrm{IRM}}(H)
$$

The negative $\Delta M$ values are usually taken as indicative of the prevalence of demagnetizing (e.g., dipole-dipole) interactions; positive values are attributed to the interactions promoting the magnetized state (e.g., direct exchange interactions). For both samples, the $\Delta M$ plots (Fig. 5) show a strong negative deviation, indicating the prevalence of magnetic dipolar interactions among the nanoparticles. ${ }^{52}$ The average reversal field, extracted from the field position of the negative peak, is higher for CFO due to the larger cobalt content $\left(\mu_{0} H_{\text {rev }} \approx 0.85 v s\right.$. $0.56 \mathrm{~T}$, for CFO and CNFO, respectively). Furthermore, the amplitude of the peak $\left(I_{\Delta M}\right)$, proportional to the interaction magnitude, ${ }^{13}$ shows again a larger value for CFO $(-0.34 v s$. -0.17$)$ (see Section 3 of $\mathrm{ESI}^{\dagger}$ for description), as expected considering the dipolar interaction energy to be roughly $E_{\text {dip }}=\left(\mu_{0} \mu^{2}\right) /\left(4 \pi d^{3}\right),{ }^{53}$ where $\mu$ is the magnetic moment of the single particle and $d$ is the distance between the particles' center (considered as the point dipole). Due to similar average particle sizes and center-to-center interparticle

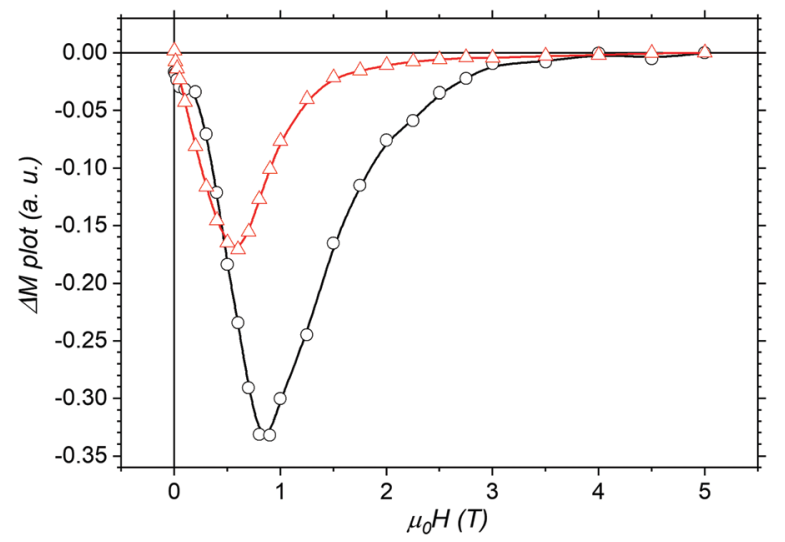

Fig. 5 The $\Delta M$-plots of samples CFO (black circles) and CNFO (red triangles) are reported. The solid lines are a guide to the eye. distances $(d)$ in the two samples, the higher saturation magnetization of CFO's particles leads to stronger dipolar interactions with respect to CNFO, being $E_{\mathrm{dip}} / k_{\mathrm{B}} \approx 44$ vs. $12 \mathrm{~K}$, respectively. This result is at odds with a very similar ZFC peak $\left(T_{\max }\right)$ and $T^{*}$, observed for the two samples.

The $M$ vs. $H$ curves evidence quite different anisotropy energies for the two samples, but similar blocking energies emerge from the $\left(M_{\mathrm{FC}}-M_{\mathrm{ZFC}}\right)$ curves measured at low field (2.5 $\mathrm{mT}$ ), and Mössbauer spectra measured with no applied magnetic field. Such results suggest the presence of a collective behavior induced by interactions, which creates a condition of higher effective anisotropy, in particular for CNFO, but which manifests only under low applied field; on the other hand, the high field applied in $M(H)$ curves suppresses the coupling and lets the single particle anisotropy dominate.

To understand this complex behavior, we applied the modified random anisotropy model. For both samples, $T^{*}$ was measured from $\mathrm{d}\left(M_{\mathrm{FC}}-M_{\mathrm{ZFC}}\right) / \mathrm{d} T$ curves at several applied magnetic fields, and the values of $T^{*}(H)$ were fitted according to eqn (10) (Fig. 6a and data summarized in Table 3). In a condition of zero applied field, the correlation length calculated from (3) is larger than the single particle diameter for both samples, but only for sample CNFO this distance is long enough to enclose two full particles $\left(L_{2 \mathrm{NP}}\right)$, considering also the organic coating (Table 3). It is worth mentioning that the correlation length in these two samples is considerably shorter than in the RCP8 sample, as expected due to the higher particle anisotropy in CFO and CNFO. A similar situation is present even at an applied field of $2.5 \mathrm{mT}$, supporting the theory of a larger effective anisotropy visible for CNFO in $M(T)$ measurements at low field and in Mössbauer spectra recorded in zero field. Indeed, at an applied field of $2.5 \mathrm{mT}$, the effective correlated volume of the magnetic material calculated from (6) for CFO and CNFO is $\approx 180$ and $740 \mathrm{~nm}^{3}$, respectively. Despite the different single particle anisotropies, the average anisotropy energy within these two volumes is comparable, about $7.2(1) \times 10^{-20} \mathrm{~J}$, which translates to a similar experimental $T^{*}$. In this framework, CFO shows stronger dipolar interactions, but the higher single particle anisotropy prevents
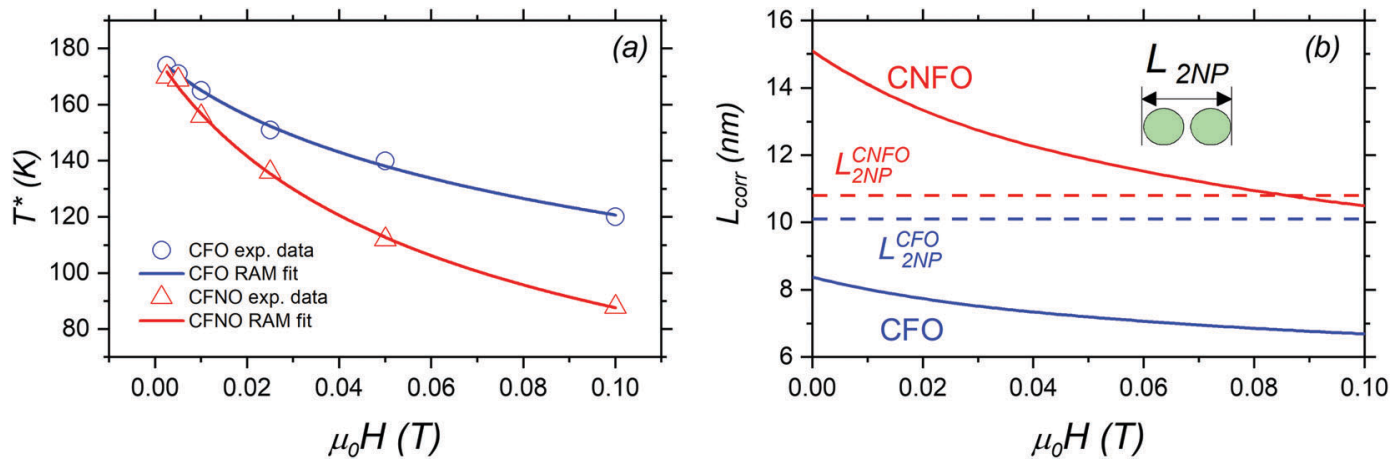

Fig. 6 Panel (a) shows the dependence of $T^{*}$ with respect to the applied field for samples CFO (blue circles) and CNFO (red triangles), with the RAM fits represented as solid lines. Error bars on the experimental points are smaller than the dots. For each sample, the correlation length $\left(L_{\text {corr }}\right)$ dependence from the applied magnetic field is reported in panel $(b)$, with the respective limit lengths which enclose two nanoparticles $\left(L_{2 N \mathrm{NP}}\right)$ represented as dashed lines. 
the formation of large correlated clusters of particles. On the other hand, CNFO, despite showing reduced dipolar interactions with respect to $\mathrm{CFO}$, is characterized by a lower NP anisotropy, which in turn allows for higher correlation lengths, at least for low enough applied fields. It is interesting to note that for CNFO, the $\mu_{0} H_{\text {limit }}$, the applied field which reduces the correlation length below the limit of $L_{2 \mathrm{NP}}$, is about $0.09 \mathrm{~T}$ (Fig. 6b). This explains why the effective cluster anisotropy is visible only at low field, indeed analyses of $M(H)$ and $\Delta M$-plot employ fields higher than $\mu_{0} H_{\text {limit }}$, which reduce to zero the correlation length, thus leading to the dominant effect of the single particle anisotropy in both samples. It is well known that dipolar interactions affect the $M(H)$ loop at low temperature (below blocking) by reducing the $H_{\mathrm{C}}$ magnitude and leading to a concomitant lower remanence with respect to the corresponding isolated particles. ${ }^{26,54}$ Anyway, in CFO (stronger interactions), $H_{\mathrm{C}}$ is greater than in CNFO due to the suppression of interparticle interaction effect caused by the much higher local single particle anisotropy. In strongly interacting particle systems, where $T_{\max }$ in the ZFC curve reflects the phase transition to a collective superspin glass state, theoretical analysis ${ }^{55,56}$ and subsequent experimental works ${ }^{25,46}$ have suggested that $T_{\max }$ is the result of interactions. Here we demonstrate for the first time that the individual particles' anisotropy plays an additional opposite role, indeed higher NP energy barriers moderate the interparticle correlations and tend to reduce the glassy temperature. Thus, the overall magnetic behavior is the balance of the interplay between the single particle anisotropy and the interparticle interactions.

\section{Conclusions}

The effect of interparticle interactions in close-packed ensembles of magnetic nanoparticles was interpreted by means of a random anisotropy model. In the first stage, the effect of strongly interacting particles in a dense ensemble was analyzed, thus explaining the origin of their SSG states by quantifying the correlation length. In addition, the method was demonstrated to be effective in order to estimate the contribution of the single particle anisotropy energy, even if it is hidden by the strong collective behavior of the sample.

The second part of the work analyzed the complex interplay of single particle magnetic anisotropy and interparticle interactions in two ensembles of particles with the same average size: pure cobalt ferrite $\left(\mathrm{CFO}, \mathrm{CoFe}_{2} \mathrm{O}_{4}\right)$ and half-substituted cobalt-nickel ferrite (CNFO, $\mathrm{Co}_{0.5} \mathrm{Ni}_{0.5} \mathrm{Fe}_{2} \mathrm{O}_{4}$ ). The lowtemperature hysteresis loops showed a strong reduction of magnetic anisotropy upon introducing nickel in place of cobalt. On the other hand, the thermally activated switching process of the magnetization analyzed by $\mathrm{ZFC}, \mathrm{FC}$ and $\left(M_{\mathrm{FC}}-M_{\mathrm{ZFC}}\right)$ protocols with a low applied field of $2.5 \mathrm{mT}$, as well as by zero-field Mössbauer spectroscopy at $300 \mathrm{~K}$, underlined a different picture, where almost the same average anisotropy energy emerged for the two samples. The interplay between the single particle anisotropy and the interparticle interactions is the key point to understand this intriguing behavior. Upon interpretation of the results by means of the random anisotropy model, it was shown that a strong single particle anisotropy prevailed in CFO. On the other hand, the right mix between the lower anisotropy and the quite long-range dipolar interactions produced a more correlated state in CNFO, which exhibited a blocking temperature higher than expected. This correlation was reduced by the application of an external field; indeed applied high fields allowed the single particle anisotropy to emerge. These results provide experimental evidence that the overall magnetic behavior of strongly interacting particles depends on the complex interplay between the single particle anisotropy energy and the interparticle interaction energy, with higher individual NP energy barriers playing against the collective behavior.

These conclusions are of interest not only from a fundamental point of view but also for their potentiality in technological applications. As demonstrated by the results of CFO and CNFO, it is possible to develop systems in which the thermal and the field-induced reversal of the magnetization can be tuned independently, opening new interesting perspectives in order to design materials for specific applications.

\section{Author's contribution}

DP and GM led the research. GM and SSL synthesized the nanoparticles. GM, RM and DP elaborated the model. CC and AM performed and analyzed the TEM measurements. GC and GM performed and analyzed Mössbauer spectra. JDT and RM performed the magnetization measurements on the RCP8/S17 systems, and SL, AMT and CS performed the magnetization measurements on the CFO and CNFO samples. MNAF revised the manuscript providing a contribution to the elaboration of the model.

\section{Conflicts of interest}

There are no conflicts of interest to declare.

\section{Acknowledgements}

G. Muscas gratefully acknowledges the Sardinia Regional Government for the financial support of his PhD scholarship (P. O. R. Sardegna F. S. E. Operational Program of the Autonomous Region of Sardinia, European Social Fund 2007-2013Axis IV Human Resources, Objective 1.3, Line of Activity 1.3.1.). G. M. and R. M. thank the Swedish Research Council (VR) for financial support. The financial support of the bilateral project CNR-CNPq 2014-15 is also gratefully acknowledged. J. A. De Toro acknowledges financial support from the Spanish Ministerio de Economía y Competitividad (MAT2015-65295-R). D. P. thanks T. A. P. for inspiring discussions. 


\section{References}

1 M. Sugimoto, The past, present, and future of ferrites, J. Am. Ceram. Soc., 1999, 82, 269-280.

2 C. R. Vestal and Z. J. Zhang, Effects of Surface Coordination Chemistry on the Magnetic Properties of $\mathrm{MnFe}_{2} \mathrm{O}_{4}$ Spinel Ferrite Nanoparticles, J. Am. Chem. Soc., 2003, 125, 9828-9833.

3 X. Batlle, N. Pérez, P. Guardia, O. Iglesias, A. Labarta, F. Bartolomé, L. M. García, J. Bartolomé, A. G. Roca, M. P. Morales and C. J. Serna, Magnetic nanoparticles with bulklike properties (invited), J. Appl. Phys., 2011, 109, $07 \mathrm{~B} 524$.

4 G. Muscas, G. Concas, C. Cannas, A. Musinu, A. Ardu, F. Orrù, D. Fiorani, S. Laureti, D. Rinaldi, G. Piccaluga and D. Peddis, Magnetic Properties of Small Magnetite Nanocrystals, J. Phys. Chem. C, 2013, 117, 23378-23384.

5 C. Sun, J. S. H. Lee and M. Zhang, Magnetic nanoparticles in MR imaging and drug delivery, Adv. Drug Delivery Rev., 2008, 60, 1252-1265.

6 M. Andrés Vergés, R. Costo, A. G. Roca, J. F. Marco, G. F. Goya, C. J. Serna and M. P. Morales, Uniform and water stable magnetite nanoparticles with diameters around the monodomain-multidomain limit, J. Phys. D: Appl. Phys., 2008, 41, 134003.

7 P. Guardia, N. Pérez, A. Labarta and X. Batlle, Controlled synthesis of iron oxide nanoparticles over a wide size range, Langmuir, 2010, 26, 5843-5847.

8 C. Singh, A. Goyal and S. Singhal, Nickel doped cobalt ferrite nanoparticles: efficient catalysts for the reduction of nitroaromatic compounds and photo-oxidative degradation of toxic dyes, Nanoscale, 2014, 6, 7959-7970.

9 A. Hannour, D. Vincent, F. Kahlouche, A. Tchangoulian, S. Neveu and V. Dupuis, Self-biased cobalt ferrite nanocomposites for microwave applications, J. Magn. Magn. Mater., 2014, 353, 29-33.

10 E. C. Stoner and E. P. Wohlfarth, A mechanism of magnetic hysteresis in heterogeneous alloys, Magn. IEEE Trans., 1991, 27, 3475-3518.

11 D. Peddis, in Magnetic Nanoparticle Assemblies, ed. K. N. Trohidou, Pan Stanford Publishing, 2014, vol. 7, pp. 159-198.

12 S. Bedanta and W. Kleemann, Supermagnetism, J. Phys. D: Appl. Phys., 2009, 42, 013001.

13 D. Peddis, C. Cannas, A. Musinu, A. Ardu, F. Orrù, D. Fiorani, S. Laureti, D. Rinaldi, G. Muscas, G. Concas and G. Piccaluga, Beyond the Effect of Particle Size: Influence of $\mathrm{CoFe}_{2} \mathrm{O}_{4}$ Nanoparticle Arrangements on Magnetic Properties, Chem. Mater., 2013, 25, 2-10.

14 D. Fiorani and D. Peddis, Understanding dynamics of interacting magnetic nanoparticles: from the weak interaction regime to the collective superspin glass state, J. Phys.: Conf. Ser., 2014, 521, 012006.

15 J.-O. Andersson, C. Djurberg, T. Jonsson, P. Svedlindh and P. Nordblad, Monte Carlo studies of the dynamics of an interacting monodispersive magnetic-particle system, Phys. Rev. B: Condens. Matter Mater. Phys., 1997, 56, 13983-13988.

16 P. Nordblad, Competing interaction in magnets: the root of ordered disorder or only frustration?, Phys. Scr., 2013, 88, 058301.
17 S. Bedanta, T. Eimüller, W. Kleemann, J. Rhensius, F. Stromberg, E. Amaladass, S. Cardoso and P. Freitas, Overcoming the Dipolar Disorder in Dense CoFe Nanoparticle Ensembles: Superferromagnetism, Phys. Rev. Lett., 2007, 98, 10-13.

18 A. A. Timopheev, S. M. Ryabchenko, V. M. Kalita, A. F. Lozenko, P. A. Trotsenko, V. A. Stephanovich, A. M. Grishin and M. Munakata, The influence of intergranular interaction on the magnetization of the ensemble of oriented Stoner-Wohlfarth nanoparticles, J. Appl. Phys., 2009, 105, 083905.

19 V. Thiruvengadam and S. Vitta, Interparticle interactions mediated superspin glass to superferromagnetic transition in Ni-bacterial cellulose aerogel nanocomposites, J. Appl. Phys., 2016, 119, 244312.

20 D. Peddis, P. E. Jonsson, S. Laureti and G. Varvaro, in Nanomagnetism: Fundamentals and Applications, ed. C. Binns, Elsevier, 2014, pp. 129-179.

21 A. G. Roca, J. F. Marco and C. J. Serna, Effect of Nature and Particle Size on Properties of Uniform Magnetite and Maghemite Nanoparticles, J. Phys. Chem. C, 2007, 18577-18584.

22 J. Chatterjee, Y. Haik and C. J. Chen, Size dependent magnetic properties of iron oxide nanoparticles, J. Magn. Magn. Mater., 2003, 257, 113-118.

23 G. Muscas, N. Yaacoub, G. Concas, F. Sayed, R. Sayed Hassan, J. M. Greneche, C. Cannas, A. Musinu, V. Foglietti, S. Casciardi, C. Sangregorio and D. Peddis, Evolution of the magnetic structure with chemical composition in spinel iron oxide nanoparticles, Nanoscale, 2015, 7, 13576-13585.

24 D. Carta, M. F. Casula, A. Falqui, D. Loche, G. Mountjoy, C. Sangregorio and A. Corrias, A Structural and Magnetic Investigation of the Inversion Degree in Ferrite Nanocrystals $\mathrm{MFe}_{2} \mathrm{O}_{4}(\mathrm{M}=\mathrm{Mn}, \mathrm{Co}, \mathrm{Ni}), J$. Phys. Chem. C, 2009, 113, 8606-8615.

25 J. A. De Toro, P. S. Normile, S. S. Lee, D. Salazar, J. L. Cheong, P. Muñiz, J. M. Riveiro, M. Hillenkamp, F. Tournus, A. Tamion and P. Nordblad, Controlled Close-Packing of Ferrimagnetic Nanoparticles: An Assessment of the Role of Interparticle Superexchange Versus Dipolar Interactions, J. Phys. Chem. C, 2013, 117, 10213-10219.

26 J. L. Dormann, D. Fiorani and E. Tronc, Advanced in Chemical Physics, Wiley, New York, 2007, vol. XCVIII, pp. 283-494.

27 D. Fiorani, J. L. Dormann, R. Cherkaoui, E. Tronc, F. Lucari, F. D’Orazio, L. Spinu, M. Nogues, A. Garcia and A. M. Testa, Collective magnetic state in nanoparticles systems, J. Magn. Magn. Mater., 1999, 196-197, 143-147.

28 O. Petracic, Superparamagnetic nanoparticle ensembles, Superlattices Microstruct., 2010, 47, 569-578.

29 X. Chen, S. Bedanta, O. Petracic, W. Kleemann, S. Sahoo, S. Cardoso and P. P. Freitas, Superparamagnetism versus superspin glass behavior in dilute magnetic nanoparticle systems, Phys. Rev. B: Condens. Matter Mater. Phys., 2005, 72, 214436. 
30 G. Herzer, in Properties and Applications of Nanocrystalline Alloys from Amorphous Precursors SE - 2, ed. B. Idzikowski, P. Švec and M. Miglierini, Springer Netherlands, 2005, vol. 184, pp. 15-34.

31 R. Alben, J. J. Becker and M. C. Chi, Random anisotropy in amorphous ferromagnets, J. Appl. Phys., 1978, 49, 1653.

32 A. Michels, R. Viswanath, J. Barker, R. Birringer and J. Weissmüller, Range of Magnetic Correlations in Nanocrystalline Soft Magnets, Phys. Rev. Lett., 2003, 91, 267204.

33 J. C. Denardin, W. C. Nunes and M. Knobel, Effects of magnetic interparticle coupling in the blocking temperature of granular Co multilayers, Phys. B, 2006, 384, 290-293.

34 W. C. Nunes, L. M. Socolovsky, J. C. Denardin, F. Cebollada, A. L. Brandl and M. Knobel, Role of magnetic interparticle coupling on the field dependence of the superparamagnetic relaxation time, Phys. Rev. B: Condens. Matter Mater. Phys., 2005, 72, 212413.

35 M. Knobel, W. C. Nunes, H. Winnischofer, T. C. R. Rocha, L. M. Socolovsky, C. L. Mayorga and D. Zanchet, Effects of magnetic interparticle coupling on the blocking temperature of ferromagnetic nanoparticle arrays, J. Non-Cryst. Solids, 2007, 353, 743-747.

36 K. Suzuki and J. Cadogan, Random magnetocrystalline anisotropy in two-phase nanocrystalline systems, Phys. Rev. B: Condens. Matter Mater. Phys., 1998, 58, 2730-2739.

37 J. Weissmüller, A. Michels, J. Barker, A. Wiedenmann, U. Erb and R. Shull, Analysis of the small-angle neutron scattering of nanocrystalline ferromagnets using a micromagnetics model, Phys. Rev. B: Condens. Matter Mater. Phys., 2001, 63, 1-18.

38 M. Knobel and W. Nunes, Superparamagnetism and other magnetic features in granular materials: a review on ideal and real systems, J. Nanosci. Nanotechnol., 2008, 8, 1880-1885.

39 K. Ridier, B. Gillon, G. Chaboussant, L. Catala, S. Mazérat, E. Rivière and T. Mallah, Individual-collective crossover driven by particle size in dense assemblies of superparamagnetic nanoparticles, Eur. Phys. J. B, 2017, 90, 77.

40 B. Pacakova, A. Mantlikova, D. Niznansky, S. Kubickova and J. Vejpravova, Understanding particle size and distance driven competition of interparticle interactions and effective single-particle anisotropy, J. Phys.: Condens. Matter, 2016, 28, 206004.

41 H. Mamiya, M. Ohnuma, I. Nakatani and T. Furubayashim, Extraction of blocking temperature distribution from zerofield-cooled and field-cooled magnetization curves, IEEE Trans. Magn., 2005, 41, 3394-3396.

42 I. J. Bruvera, P. Mendoza Zélis, M. Pilar Calatayud, G. F. Goya and F. H. Sánchez, Determination of the blocking temperature of magnetic nanoparticles: the good, the bad, and the ugly, J. Appl. Phys., 2015, 118, 184304.

43 J. A. De Toro, S. S. Lee, D. Salazar, J. L. Cheong, P. S. Normile, P. Muñiz, J. M. Riveiro, M. Hillenkamp,
F. Tournus, A. Tamion and P. Nordblad, A nanoparticle replica of the spin-glass state, Appl. Phys. Lett., 2013, 102, 183104.

44 J. I. Gittleman, B. Abeles and S. Bozowski, Superparamagnetism and relaxation effects in granular $\mathrm{Ni}^{-\mathrm{SiO}_{2}}$ and $\mathrm{Ni}$ $\mathrm{Al}_{2} \mathrm{O}_{3}$ films, Phys. Rev. B: Condens. Matter Mater. Phys., 1974, 9, 3891-3897.

45 P. S. Normile, M. S. Andersson, R. Mathieu, S. S. Lee, G. Singh and J. A. De Toro, Demagnetization effects in dense nanoparticle assemblies, Appl. Phys. Lett., 2016, 109, 152404.

46 M. S. Andersson, R. Mathieu, S. S. Lee, P. S. Normile, G. Singh, P. Nordblad and J. A. De Toro, Size-dependent surface effects in maghemite nanoparticles and its impact on interparticle interactions in dense assemblies, Nanotechnology, 2015, 26, 475703.

47 Q. Song and Z. J. Zhang, Correlation between spin-orbital coupling and the superparamagnetic properties in magnetite and cobalt ferrite spinel nanocrystals, J. Phys. Chem. B, 2006, 110, 11205-11209.

48 L. Kumar, P. Kumar and M. Kar, Cation distribution by Rietveld technique and magnetocrystalline anisotropy of $\mathrm{Zn}$ substituted nanocrystalline cobalt ferrite, J. Alloys Compd., 2013, 551, 72-81.

49 E. Fantechi, G. Campo, D. Carta, A. Corrias, C. de Julián Fernández, D. Gatteschi, C. Innocenti, F. Pineider, F. Rugi and C. Sangregorio, Exploring the Effect of Co Doping in Fine Maghemite Nanoparticles, J. Phys. Chem. C, 2012, 116, 8261-8270.

50 E. P. Wohlfarth, Relations between Different Modes of Acquisition of the Remanent Magnetization of Ferromagnetic Particles, J. Appl. Phys., 1958, 29, 595-596.

51 P. Kelly, K. O’Grady and P. Mayo, Switching mechanisms in cobalt-phosphorus thin films, IEEE Trans., 1989, 25, 3881-3883.

52 J. A. De Toro, M. Vasilakaki, S. S. Lee, M. S. Andersson, P. S. Normile, N. Yaacoub, P. Murray, E. H. Sánchez, P. Muñiz, D. Peddis, R. Mathieu, K. Liu, J. Geshev, K. N. Trohidou and J. Nogués, Remanence plots as a probe of spin disorder in magnetic nanoparticles, Chem. Mater., 2017, 29, 8258-8268.

53 S. Mørup, M. F. Hansen and C. Frandsen, Magnetic interactions between nanoparticles, Beilstein J. Nanotechnol., 2010, 1, 182-190.

54 D. Kechrakos and K. N. Trohidou, Competition between dipolar and exchange interparticle interactions in magnetic nanoparticle films, J. Magn. Magn. Mater., 2003, 262, 107-110.

55 S. Mørup, Superparamagnetism and Spin Glass Ordering in Magnetic Nanocomposites, Europhys. Lett., 1994, 28, 671-676.

56 M. F. M. Hansen and S. Mørup, Models for the dynamics of interacting magnetic nanoparticles, J. Magn. Magn. Mater., 1998, 184, L262-L274. 